\title{
Fundaciones. Beatriz Sarlo en el siglo XIX*
}

Foundations. Beatriz Sarlo in the 19th Century

Alejandra Laera ${ }^{\mathrm{a}}$

Universidad de Buenos Aires, Argentina

alelaera@gmail.com

ORCID: https://orcid.org/0000-0003-4691-8661

DOI: https://doi.org/10.11144/Javeriana.cl24.bses

\section{Resumen:}

Este ensayo sistematiza los abordajes de la literatura argentina del siglo XIX hechos por Beatriz Sarlo en función de un proyecto crítico más amplio, en el que ha privilegiado el siglo XX y en particular sus primeras décadas, y que ha desarrollado por lo menos hasta el presente siglo. La hipótesis central es que la insistencia en analizar el momento fundacional de la literatura argentina y la obra y figura de escritores como Sarmiento y Echeverría, se debe al objetivo de encontrar un primer momento modernizador previo a la modernidad cultural de los años que investigó en su ya clásico Una modernidad periférica. Buenos Aires 1920 y 1930 (1988). Asimismo, el ensayo propone pensar el estudio inicial de Sarlo dedicado a Juan María Gutiérrez en relación con una historia de la literatura narrada desde una perspectiva moderna, pero también con su propia posición como crítica.

Palabras clave: Sarlo, romanticismo, modernidad, historia literaria.

\section{Abstract:}

This essay systematizes Beatriz Sarlo's nineteenth-century Argentinean literature approaches, which are part of a broader critical project focused on the twentieth century, particularly on the first decades, that she has extended at least to the present century. The central hypothesis is that Sarlo's insistence on examining the founding moment of Argentinean literature and the work and figure of writers such as Sarmiento and Echeverría is due to her intention to find a first modernizing moment before the cultural modernity she observes in her classic work Una modernidadperiférica. Buenos Aires 1920 y 1930 (1988). Furthermore, the essay aims to explore Sarlo's initial study about Juan María Gutiérrez in relation to a literary history narrated from a modern perspective but also concerning her position as a critic.

Keywords: Sarlo, romanticism, modernity, literary history.

Cuando en 2007 Beatriz Sarlo publica Escritos sobre literatura argentina, una compilación de sus notas, artículos y ponencias no recogidos en libro hasta el momento, se hace ostensible el lugar que la literatura del siglo XIX argentino ocupa en el conjunto de su producción crítica. De un total de casi setenta textos, aquellos dedicados al XIX son solo cuatro y están incluidos en una sección que, a diferencia de las otras tres, no lleva por título una referencia cronológica. La sección abre el volumen y se titula "Ser escritor, ser argentino, ser porteño" ${ }^{1}$. Más allá de que la organización y el orden no respondan por completo a Sarlo, al tratarse de una cuidadísima edición a cargo de Sylvia Saítta, son varias las observaciones que me interesa hacer a partir de este volumen y a la luz de esa suerte de prólogo que firma Sarlo y sirve de marco a los artículos.

La primera observación es que los textos incluidos datan recién de los años ochenta, ya que su autora eligió dejar afuera aquellos correspondientes a un periodo inicial que abarca el final de los sesenta y la década del setenta. La segunda es que los años ochenta, justamente, parecen concentrar su interés en el siglo XIX, sobre el que apenas hay un ensayo posterior. La tercera es que los cuatro escritos que toman el XIX tratan sobre Domingo F. Sarmiento o sobre sus libros. Y la última apunta a la decisión de reordenar esos escritos sin atenerse estrictamente a la cronología, de modo que la nueva organización le otorga a cada uno un sentido particular respecto de los demás. De entrada, vemos que la década del ochenta evidencia una inflexión en la

Notas de autor 
producción crítica de Sarlo pero también que el corte no solo resulta crucial respecto del conjunto de su obra, sino en particular respecto del siglo XIX. Es que ya a fines de los sesenta, y además de dos fascículos sobre los neorrománticos de la segunda mitad del siglo redactados en 1967 para la edición original de la Historia de la literatura argentina del Centro Editor de América Latina, Sarlo había publicado un primer y único libro que estaba dedicado a la figura de Juan María Gutiérrez, integrante de la generación romántica del 37 e iniciador de las tareas sistemáticas de investigación en literatura argentina.

Ese libro, por lo tanto, que una joven crítica que aún firma Beatriz Sarlo Sabajanes escribe antes de lo que sería su clásico Buenos Aires: modernidad periférica (1988), está borrado de su pasado, como si ella misma hubiera decidido empezar su trayectoria crítica recién en los años ochenta. Por un lado: tras sus inicios juveniles de un modo tan promisorio como institucionalizado, ya que el libro sobre Gutiérrez, escrito en 1967 a los 26 años, ganó el premio del Fondo Nacional de las Artes ${ }^{2}$. Y por otro lado: también después de su periodo de escritura filomilitante, marcado por su fuerte compromiso con la política y sus intentos por articularla con la crítica a través del periodismo cultural ${ }^{3}$. Así es como queda atrás, y más lejos incluso de lo que resultaría por una cuestión de fechas, este libro sobre Gutiérrez que al estudiar la obra de Sarlo es tenido en cuenta, en los mejores casos, como rareza o vago precedente.

En su mirada retrospectiva de finales de los años 2000, ya ocupando una incuestionable posición canónica dentro de la crítica cultural argentina y con reconocimiento internacional en el ámbito de los estudios literarios, el gesto de Sarlo implica, para el siglo XIX, que a los cuatro textos incluidos en Escritos de literatura argentina habría que sumar solo lo que escribió a partir de 1980 para tener completa su obra autorizada. Ante todo: el artículo sobre Recuerdos de provincia en coautoría con Carlos Altamirano en ese mismo año de 1980, que publicaron entonces en una revista venezolana e incluyeron en el volumen Ensayos argentinos. De Sarmiento a la vanguardia, cuya primera edición es de 1983; y el prólogo a la Obra escogida de Esteban Echeverría, también con Altamirano, publicado en la Biblioteca Ayacucho en 1991 ${ }^{4}$. Y además: su colaboración entre 1980 y 1981, retomando aquella no autorizada de finales de los sesenta, en la edición aumentada de la Historia de la literatura argentina del Centro Editor de América Latina, con dos fascículos sobre José Hernández y Martín Fierro escritos con María Teresa Gramuglio, que se complementó con el volumen Martín Fierro y su crítica que hicieron para la colección Capítulo.

El conjunto es escueto, sobre todo en contraste con la prolífica producción de Sarlo, pero a la vez es potente, está concentrado en poco más de una década, y se convirtió en una contribución canónica para reflexionar sobre el siglo XIX, puntualmente sobre los románticos rioplatenses. En gran medida ello se debe a la coherencia de los planteos vinculados con la fundación de la nación, a la insistencia en ciertos puntos o problemas culturales como la construcción de la figura de escritor, a la posibilidad de inferir, de ese conjunto, una idea muy moderna de la literatura y una concepción de la labor crítica y de la figura del crítico que apostó a la investigación rigurosa sin ceder ni la creatividad propia del ensayismo ni la intervención propia del intelectual. La reordenación de los Escritos de literatura argentina, particularmente de esa primera parte que incluye los textos sobre el XIX, va en esa dirección: empieza con tres lecturas sobre Sarmiento, y hace de la cuarta, que abre con Echeverría y su impulso fundacional, el nexo con todas las lecturas del siglo XX y de la contemporaneidad. Se diría que el corte se practica, justamente, en esos años iniciales de la década de 1980 que marcan un doble umbral: en el plano de la política nacional preparan el camino para el retorno de la democracia en 1983; en el plano de la trayectoria individual, constituyen una suerte de periodo preacadémico, anterior al ingreso como profesora regular de literatura argentina del siglo XX en la Universidad de Buenos Aires, y en el que predomina el trabajo en colaboración, dentro del cual la formación de la revista cultural Punto de Vista vertebrará las siguientes dos décadas de su labor intelectual.

Por todo esto, propongo leer esas intervenciones sobre el siglo XIX como plataforma para pensar el resto de la producción crítica de Sarlo sobre literatura. Como si el estudio compacto y puntual del XIX le sirviera para, partiendo de allí y dejándolo atrás, abordar plenamente el XX. Como si hubiera buscado en el romanticismo las bases de un proyecto frustrado de modernización literario cultural para poder llegar después 
a su realización actualizada en la década de 1920, a la que se dedicará con insistencia, igual que a muchos de los núcleos de modernidad que recorren el transcurso del siglo XX y estallan en el XXI.

\section{Los románticos, para pensar una nación moderna}

¿Qué lee Sarlo en Sarmiento? No tanto la consabida oposición entre la civilización y la barbarie para interpretar la realidad sudamericana, ni la idea del viaje como posibilidad de ingresar a un mundo moderno y como práctica de apropiación; tampoco la figura del publicista que busca producir hechos con las palabras, ni la del biógrafo fascinado con el objeto de su narración. Antes que nada, y aun cuando todo aquello está en el telón de fondo, lo que lee es el impulso autobiográfico como vía para la construcción de la nación. Y junto con Altamirano, en esa lectura en coautoría, ambos desmontan así todo lo que, en Recuerdos de provincia, permite unir "historia nacional y biografía personal" (Altamirano y Sarlo, Ensayos argentinos 19). Con las herramientas que tomaron por esos años de la sociología de la cultura, pero sin ceder nada del análisis textual ni tampoco del análisis político ideológico, muestran el modo en que Sarmiento trama su relato de vida alrededor de un doble eje: por un lado, el autodidactismo, con la apuesta que implica por la educación, y por el otro, la descendencia, que en su caso supone tanto un linaje colonial como patrio. En el cruce entre la "carrera del mérito" - personal - y la "herencia" - familiar-, Sarmiento habría diseñado en Recuerdos de provincia, según la hipótesis elaborada por Sarlo y Altamirano, toda una candidatura política echando mano de los recursos de la literatura. De esta manera, a su vez, los propios autores consiguen ligar literatura y política y, reactualizando en su lectura el postulado romántico que Sarmiento cifra en Facundo, encuentran en la construcción de su vida una alegorización de la construcción de la nación.

El artículo sobre Sarmiento, que potencia los intereses conjuntos de sus dos autores, se edita varias veces por esos años y también después. Junto con la mencionada primera edición en la revista Escritura de Caracas (1980), publican una versión más breve en Punto de Vista (“Identidad, linaje y mérito de Sarmiento", 1980). Tres años más tarde, cuando se incluya, con el título "Una vida ejemplar: la estrategia de Recuerdos de provincia”, en Ensayos argentinos. De Sarmiento a las vanguardias, publicado por el Centro Editor de América Latina, el eje de lo nacional resulta clave para ligar la hipótesis sobre Sarmiento con las preguntas sobre el nacionalismo que se hizo la vanguardia en la segunda década del siglo XX y que Sarlo aborda en el texto que cierra el volumen. Dos detalles resultan fundamentales en esa ocasión para pensar las condiciones de enunciación del libro: la dedicatoria general a Boris Spivacow, el artífice del Centro Editor de América Latina, porque gracias a él trabajaron durante la dictadura, entre 1976 y 1983 , y la dedicatoria del artículo a David Viñas, afiliándose así a su mirada político-ideológica y a la línea crítica de lo que fuera tres décadas antes la revista Contorno de la que fue uno de los creadores. Cuando el artículo vuelva a salir en la segunda edición de Ensayos argentinos que se publica, ampliada y con algunos cambios, bajo el sello Ariel en 1997, ya no incluye la dedicatoria a Viñas, pero mantiene la dedicatoria general a Spivacow y agrega un nuevo prólogo, esta vez conjunto, donde se aclara que los textos fueron publicados previamente tanto en Punto de Vista como en revistas del exterior dirigidas, entre otros, por Ángel Rama y Antonio Cornejo Polar, quienes por entonces, cabe agregar, buscaban formar una red de intervención crítica que desde América Latina se proyectaba a toda la región y también a la academia norteamericana. El agradecimiento permite entender la circulación a corto y mediano plazo de estos textos ensayísticos y académicos, a la vez que, junto con los cambios, permite constatar cuáles son los intereses sostenidos en el tiempo. La tercera y última edición de Ensayos argentinos es publicada en 2016 por Siglo XXI, editorial en la que Sarlo y Altamirano vienen sacando sus libros por lo menos en los últimos quince años, y recupera intacta a la anterior de 1997.

A lo largo de esos años y en paralelo a la circulación de este artículo, el interés en la figura fundacional de Sarmiento encuentra su complemento en un artículo, también en coautoría con Altamirano, sobre Esteban Echeverría, solo que esta vez la distribución de las partes se hace explícita: mientras Altamirano firma aquella 
dedicada a la política y las ideas ("El doctrinario"), Sarlo firma la dedicada a la figura de escritor y a su literatura ("El poeta"). Tras la primera publicación como prólogo de la Obra escogida de Esteban Echeverría en 1991, el artículo fue incluido, precisamente, en la edición ampliada de los Ensayos argentinos, en 1997 y en $2016^{5}$.

En "El poeta", Sarlo entrega un relato de la trayectoria intelectual de Esteban Echeverría: desde el viaje a Europa y el conocimiento del romanticismo a las primeras producciones poéticas y su efecto en la escena literario-cultural de la época en el Río de la Plata. Echeverría, señala Sarlo anticipando el viraje impuesto por el exilio de finales de la década del treinta y hasta la muerte en Montevideo, pudo conocer en ese viaje iniciático a Europa la "doble investidura de ideólogo y poeta" del escritor romántico (Altamirano y Sarlo "El poeta"). Ahora bien: es en este artículo donde Sarlo propone, a propósito de Echeverría, una lectura del romanticismo que permite leer a todos los integrantes de la Generación del 37 y releer, también, su propio abordaje de Sarmiento. Me refiero a la idea de que los románticos del 37, en su ruptura total con la tradición colonial y en la distancia respecto del modelo unitario de importación cultural, deben fundar una cultura nacional en el "desierto", deben construirla "a partir de cero". Aunque no llegue a definirlo ni a explicar su composición, el "desierto", tal como lo piensa Sarlo, supone esa mezcla de tradiciones disponibles que un estudio detenido nos permite precisar y en el que están incluidas las crónicas de la conquista del Río de la Plata, los relatos de los viajeros ingleses de los años veinte, las novelas de René de Chateaubriand y de James Fenimore Cooper, así como un modo de nombrar la amplia extensión de tierra habitada por los indios. Esa noción de "desierto", entonces, a la que los románticos recurren hasta su naturalización total, es utilizada por Sarlo para intensificar una metáfora fundacional: el "desierto" no sería, así, solo del orden del territorio, sino también de las instituciones y de la cultura. Ese "desierto", nombrado y descripto por Echeverría en "La cautiva” (1837), pero también después por Sarmiento en Facundo (1845), entre otros, habilitaba a su vez la idea de vacío y, por lo tanto, la misión fundadora de los románticos. De allí las múltiples implicancias de la pregunta que formula Sarlo para pensar la producción de la Generación del 37: “¿cómo expresar literariamente una cultura y una sociedad que se juzga necesario fundar?” ("El poeta” 26).

Como puede verse, el ensayo sobre Echeverría, con su reflexión acerca del gesto fundador de los románticos y del modo en que ese gesto estaría renovando en todos los niveles aquello que se quiere nacional, actúa retroactivamente sobre la lectura que se había hecho de Sarmiento. Retroactivamente, en la medida en que se trata, a esta altura, de una interpretación que abarca en su conjunto el romanticismo clásico, según puede considerarse el de la Generación del 37, y que además encuentra en los propios letrados que la conforman, y por la doble vía que abren Echeverría y Sarmiento, a los protagonistas de esa tarea fundadora. Explica Sarlo:

No se puede subestimar la importancia de los letrados para este proyecto. Son los letrados los que pueden encontrar esas verdades concretas que la literatura deberá expresar en su traducción sensible y afectiva. Pero son también los letrados quienes hacen posible el escenario público donde esas verdades pueden exponerse. En los letrados recae, por otra parte, la responsabilidad de ser originales, y la poesía, especialmente, deberá hacerse cargo de abrir el camino en una sociedad que es nueva, pero que carga con una herencia de la que es necesario separarse para que esa novedad (y fidelidad a lo "real") se realice por completo. ("El poeta" 26)

Si en Echeverría está el postulado teórico de la misión de los letrados, en Sarmiento estaría, tal como Sarlo y Altamirano habían llegado a advertirlo un par de años antes en Recuerdos de provincia aun cuando no lo hicieran explícito, su puesta en práctica. Lo que quiero decir es que, en el ensayo sobre Echeverría, Sarlo consigue, por primera vez, ampliar el foco de la figura de escritor - ya no se trataría estrictamente ni de Gutiérrez ni de Sarmiento ni de Echeverría - para convertirlo en protagonista de un momento particular, de una circunstancia, de un conjunto de ideas y de prácticas, que lo exceden. En este caso, el romanticismo en su inflexión rioplatense. Y si Sarmiento, antes que Echeverría, será siempre la figuración culminante de ese planteo que hace del letrado el ideólogo y ejecutor de un proyecto nacional renovador, lo será en tanto expresión privilegiada de su tiempo; más aún: lo será porque es en tanto hombre de su tiempo que puede ver más allá de él y, retomando la hipótesis de Altamirano y Sarlo, candidatearse simbólica y anticipadamente como presidente en su autobiografía. 
Fundar, construir desde el vacío, hacerlo todo de nuevo: la misión del letrado, puede inferirse también, es una misión que supone una visión de mundo moderna y que implica un gesto de modernización. Se trata de fundar una nación moderna. En ese sentido, el corte que practican los románticos con el pasado es de diverso orden: por un lado, es fuertemente político, en particular con las posiciones anteriores a Mayo; por otro lado, es a la vez cultural y literario por su anticonservadurismo y su anticlasicismo, y también por su defensa de la originalidad creativa en lugar de la copia o la mera importación, lo que lo acerca a un cierto relativismo. El hecho de que este gesto de los románticos, sin embargo, termine en la confrontación total con el rosismo a partir del año 1838 pospone el proyecto de fundación de una nación moderna a largo plazo, y le da un giro a la misión de los intelectuales, que se continúa, ya de un modo diferente y con otros objetivos, en el exilio.

Por todo esto, creo, y aunque Sarlo no lo explicite ni subraye, aunque no sea el tema ni la hipótesis principal de su abordaje del XIX, el romanticismo puede leerse, a la luz de su producción posterior y de su perspectiva sobre la política y la cultura, como una clave inicial y preemergente de su insistencia casi obsesiva en la búsqueda de lo moderno, en las prácticas de modernización y en la función de los intelectuales en la Argentina. Es precisamente eso lo que encontraría plenamente en la Buenos Aires de la década de 1920, en ese cruce original entre lo nacional y lo cosmopolita que hizo de la ciudad el espacio donde procesar las dimensiones políticas, sociales, literarias y culturales.

Como si dijéramos: fue en sus diversas exploraciones en el romanticismo que Sarlo llegó a proponer esa lectura ya general y definitoria a partir de la figura de Echeverría, que a su vez es lo último, prácticamente, que escribió sobre el tema y sobre el siglo XIX en su conjunto. Porque esas lecturas se remontan, como indiqué al comienzo, a finales de los años sesenta, y acompañan el libro sobre Juan María Gutiérrez. De hecho, sus contribuciones para la Historia de la literatura argentina del CEAL, entre 1967 y 1968, son sobre romanticismo: "La segunda generación romántica: Gutiérrez, Andrade" y "Los últimos románticos" — sobre Carlos Guido y Spano y Rafael Obligado-. Más todavía, cuando en este último fascículo resume rápidamente a la generación anterior menciona como romántico a José Hernández, sobre quien escribirá precisamente, en colaboración con María Teresa Gramuglio, para la segunda edición de $1980^{6}$. Es decir que no habría en toda la producción de Sarlo sobre el siglo XIX, sola o en coautoría, ningún tema que no haya estado vinculado de algún modo con el romanticismo. El lugar fundacional del romanticismo puede anticiparse, incluso, tan inesperada como fallidamente, en el comienzo de la entrega "Panorama del cuento", también para la segunda edición de la Historia de la literatura argentina de CEAL. Allí, para hablar del desarrollo del género en el siglo XX marca una "línea fracturada" que, recomponiendo el armado que propone, iría de "El matadero" a Roberto J. Payró pasando por José Hernández, y que se cruzaría con otra que va de Sarmiento a Fray Mocho. De este modo, Sarlo ubica los comienzos del cuento en el cruce del trabajo realista con la lengua y en la práctica periodística, para llegar a Payró y a Fray Mocho, dos de los escritores periodistas que ella misma convertiría en las figuras representativas del escritor profesional en la Argentina del Centenario, en el artículo sobre el campo intelectual de comienzos de siglo escrito con Carlos Altamirano e incluido en todas las ediciones de los Ensayos argentinos ${ }^{7}$.

Así, el romanticismo resulta doblemente fundacional: no solo lo es para pensar la literatura, la cultura y a los intelectuales de una nación moderna, sino también para pensar una historia de la literatura para la cual la continuidad es interrumpida, "fracturada". Pero lo es, además, en un sentido que propongo pensar casi reflexivamente: está allí también, en el romanticismo, entre esos románticos, el principio de la labor crítica. Porque es el crítico literario, en la figura de Juan María Gutiérrez, el que primero le llama la atención; es aquel que lee, historiza e interpreta: como si hubiera que despejar ante todo esa función crítica, para después borrarla y ponerse a construir otros objetos. 


\section{Juan María Gutiérrez, de la historia de la literatura a la crítica literaria}

¿Por qué quiere Sarlo olvidar este libro? ¿Es por su tema, por su organización o por su interpretación? ¿Qué es aquello que ya no la representa: un interés en particular, una manera de leer o una escritura? Si bien no sería del todo acertado dar por sabida una respuesta, sí hay algunos rasgos de Juan Maria Gutiérrez: historiador y crítico de nuestra literatura, que Sarlo publicó en 1967 con el apoyo económico del Fondo Nacional de las Artes, que lo distancian de su futura producción crítica. Por lo pronto, el libro está organizado de un modo reconociblemente académico, que sigue las pautas convencionales generalmente asociadas, sobre todo en ese entonces, a la escritura de una tesis. Tanto la división en capítulos como la secuencia en la que se presenta la investigación responden a ese modelo.

El primer capítulo describe la situación cultural en el Río de la Plata anterior al romanticismo, desde 1810, y pone el foco en el surgimiento de sociedades literarias y de la prensa periódica y en la educación. Se trata, como puede verse, de tres zonas que para Sarlo resultarían centrales en su posterior abordaje de los fenómenos literarios y que, en el libro sobre Gutiérrez, anticipan ese desplazamiento, en buena medida impulsado por la sociología de la cultura y las lecturas que haría de Raymond Williams, que va del contexto histórico político a las prácticas culturales y a los soportes materiales de la literatura. Solo que en este libro la investigación sobre los temas afines a la cultura material y la investigación sobre Gutiérrez se solapan por completo, ya que Sarlo usa como fuente al propio Gutiérrez, lo que implica un problema metodológico que la autora parece intuir:

No creo incurrir en falacia al tomar como fuente al crítico mismo que estudiaré, por dos motivos: primero porque Juan María
Gutiérrez es el primero de nuestros hombres de letras que proporciona una visión coherente de la literatura como tal y de la
injerencia de lo no-literario dentro del campo de lo literario; segundo porque no considero, en este estudio, a Gutiérrez como
poeta o narrador sino como crítico y, después de haber comprobado la información que suministra a la luz de los métodos
correspondientes, puede ser aceptada como documentación aunque sea él precisamente el objeto de esta investigación. Juan
María Gutiérrez realizó una tarea indispensable en el momento histórico que le tocó vivir, y creo que esta tarea conserva
todavía su validez como fuente de datos. (8-9)

Sin embargo, más allá de ese desliz metodológico que confunde la perspectiva crítica al oscilar entre el objeto de la investigación y las fuentes con las cuales se lo estudia, me interesa destacar cómo ya aparece un interés en las prácticas de la crítica, concentradas en este caso en Gutiérrez: sus protocolos, la elección de sus objetos, las herramientas sociológicas, la posición del especialista, su pretensión de rigurosidad.

El segundo capítulo se detiene ya estrictamente en la crítica literaria y en él Sarlo señala y explica la relación estrecha que hay entre su surgimiento y la literatura nacional. Esa relación se basa fundamentalmente, podría decirse, en dos cuestiones que reaparecen en diversos momentos del texto y que es posible advertir en la posición general de la autora. La primera es la necesidad de que haya una cantidad suficiente de escritos interesantes, que posean un cierto valor literario que va más allá de la publicación circunstancial, porque, si no, es imposible que pueda existir la crítica. La segunda, de algún modo consecuencia de la anterior, es que antes de que exista una crítica literaria propiamente dicha es preciso que pueda reconocerse una historia de la literatura nacional. Al respecto, querría destacar dos corolarios que, más allá del siglo XIX, son cuestiones importantísimas para pensar la concepción de Sarlo sobre la literatura y la crítica y que aparecen muy incipientemente acá. En principio, una suerte de discusión sobre la especificidad y la autonomía literarias de cuño moderno que permite entender tanto el hecho de que esa historia literaria empiece en el romanticismo y de ningún modo antes para la perspectiva sarliana: como si dijéramos que la lucha política a través de la literatura supone una legitimación de la literatura que es previa o complementaria a esa función política. Y además, la inclinación por una crítica literaria que usa la lectura textual como herramienta pero privilegia la contextualización histórico cultural y la atención a las prácticas involucradas: una crítica que es sociología de la cultura y también sociocrítica y que por ese camino, que la conduce finalmente a la crítica cultural, tiene una gran afinidad con la historia de la literatura. 
Los tres siguientes capítulos presentan, respectivamente, la mirada de Gutiérrez sobre ciertos conjuntos literarios: la literatura colonial, la literatura española y la literatura nacional. A lo largo de los mismos, Sarlo va dando cuenta del modo en el que Gutiérrez encuentra entre sus contemporáneos, y en contra de todo hispanismo, aquello que le interesa destacar del cambio que hay entre lo neoclásico y lo romántico. Y al hacerlo, también parece ir definiendo sus propios postulados sobre los inicios de la literatura argentina. Mientras el contraste entre los dos grandes estudios biográficos de Juan María Gutiérrez, sobre el neoclásico Juan Cruz Varela y el romántico Esteban Echeverría, le permite posicionarse respecto de dos ideas muy diferentes de literatura, el abordaje de Gutiérrez en cotejo con Alberdi y Echeverría anticipan la conexión que hará, en el ensayo sobre el poeta, de la idea de una "literatura nacional" con la idea de una literatura que "se independiza así de toda labor ancilar para convertirse ella misma en poder y motivación de cambio, y no mero apoyo o discusión de ese cambio" (132). Y si bien puede decirse que este postulado que lee Sarlo en Echeverría se frustra, tras los intentos de los años treinta, con los exilios políticos de finales de la década, también es cierto que la postergación de una suerte de autonomía es la que la lleva, por la vía del romanticismo más autofigurativo y profesionalista, al modernismo de los años de 1920 que estudió en Una modernidad periférica. Buenos Aires, 1920-1930.

\section{Corolario}

Si tuviera que definir lo que es el siglo XIX en la trayectoria de Beatriz Sarlo, diría que es la posibilidad de hacer la historia de la literatura necesaria antes de dedicarse por completo a la crítica, a la vez que de poner en juego ideas sobre la literatura y las prácticas críticas que se le fueron imponiendo sobre sus objetos más o menos circunstanciales de estudio.

Por supuesto, el punto de vista que asume Sarlo desde el vamos se avala, explícita o implícitamente, en historiadores y estudiosos de la literatura argentina. En el campo de la historia, Tulio Halperín Donghi, que realizó varias e imprescindibles incursiones en el romanticismo local: mientras su primer libro fue, de hecho, El pensamiento de Echeverría, de 1951, a comienzos de los ochenta estaba trabajando en Una nación para el desierto argentino, que publicó en 1982 y fue referencia en las exploraciones decimonónicas de Sarlo junto con Altamirano. En el campo de los estudios literarios, además de David Viñas, con su Literatura argentina y realidad política, de 1964, resultó clave otra figura de la revista Contorno, Adolfo Prieto, que ya había editado el volumen colectivo Proyección del rosismo en la literatura argentina en 1959 y cuya figura crítica se redimensionaría a mediados de los años ochenta con la publicación de El discurso criollista en la formación de la literatura argentina. En un registro menos evidente y todavía poco explorado, es considerable Bernardo Canal Feijóo, que aparece mencionado en su primer libro - del que además el escritor santiagueño fue jurado - y de quien toma su interés por el desierto y la ciudad. En la misma órbita, despertó su atención crítica, verificable sobre todo en los escritos de la década del ochenta, Ezequiel Martínez Estrada, aunque la atención que le presta como autor de Radiografía de la pampa y de La cabeza de Goliat, proviene ya de la que había despertado en los integrantes de Contorno. Como sea que hayan sido la aproximación y lectura de todos estos referentes locales, más o menos perdurables en la trayectoria de Sarlo, lo que me interesa es que prácticamente en todos los casos el romanticismo resulta un nudo crítico privilegiado, según puede verse en especial en Halperín Donghi y Viñas. Pero, además, querría recalcar que los años ochenta, en sintonía con las reflexiones sobre la idea de nación suscitadas por la coyuntura del fin de la dictadura militar con su autodenominado Proceso de Reorganización Nacional y el esperado retorno de la democracia, tienen el foco crítico puesto en un siglo XIX protagonizado por la romántica Generación del $37^{8}$. Como había declarado David Viñas al comienzo de Literatura argentina y realidad política, reeditado por el Centro Editor de América Latina en 1983, la historia de la literatura argentina es la historia de una voluntad, y por eso puede decirse que empieza con los románticos en tiempos de Rosas. 
En ese punto, el primer libro de Sarlo, junto con los demás artículos sobre el siglo XIX argentino algunos de ellos en colaboración con Carlos Altamirano y reunidos en las dos primeras ediciones de Ensayos argentinos. De Sarmiento a las vanguardias, y el resto incluidos en Escritos sobre literatura argentina-, le permitieron ingresar al siglo XIX argentino desde el romanticismo para saltar desde allí, como expliqué, al siglo XX. Pero además, así como su primer libro — iescrito casi para ser borrado! - le permitió, según también expliqué, proponer ciertos postulados sobre la literatura, sus ideas y sus prácticas, los demás ensayos sobre el siglo XIX ponen en acción no solo esas ideas sino también esas prácticas. Ya a partir de sus acercamientos al XIX se puede pensar en Sarlo la importancia de la investigación en archivos; la relevancia de la publicación en libro que saca a los artículos de la publicación circunstancial, y la necesaria intervención en el campo cultural con la crítica que surge del periodismo. Todos estos rasgos, que pueden detectarse en la trayectoria y el interés de Sarlo, se unen, creo, en su búsqueda de sistematicidad: estudiar un archivo, componer una obra, postular un horizonte crítico desde diversos frentes.

Eso mismo que se destaca en Echeverría al haber sido el primero en publicar un volumen de poemas en el Río de la Plata, se puede pensar para la crítica literaria, tan apegada a la publicación circunstancial en la prensa periódica: desde sus comienzos Sarlo apunta a un doble frente. Con su estudio sobre Juan María Gutiérrez y con los Ensayos argentinos toma distancia del periodismo y apuesta a la sistematicidad del libro o el volumen de ensayos, a la par que encara una intervención en la prensa periódica que al comienzo también tiene como objeto el siglo XIX aunque paulatinamente se corra de él. Como si la legitimación en tanto crítica literaria viniera del libro, del largo aliento de la investigación que antecede a la redacción del libro y que se complementa con la actividad académica, pero a la vez requiriera, para redimensionarse cultural y políticamente, de la creación de una revista o de la intervención en la prensa y en los medios, ya con la mirada atenta en el presente. Con ese punto de llegada, que se atisba en el inicio de los años noventa y que se afirma a lo largo de la década, ya sea desde la cátedra de literatura argentina del siglo XX, ya sea participando activamente en el debate sobre los estudios culturales latinoamericanos, ya sea con su exitoso libro de 1994 Escenas de la vida posmoderna: Intelectuales, arte y videocultura en la Argentina, el siglo XIX va quedando cada vez más atrás.

\section{Referencias}

Altamirano, Carlos y Beatriz Sarlo. Ensayos argentinos. De Sarmiento a la vanguardia. Centro Editor de América Latina, 1983.

Altamirano, Carlos y Beatriz Sarlo. "El poeta pensador". Prólogo a Esteban Echeverría, Obras escogidas. Biblioteca Ayacucho, 1991, pp. IX-LIII.

Sarlo, Beatriz. Escenas de la vida posmoderna: intelectuales, arte y videocultura en la Argentina. Ariel, 1994.

Sarlo, Beatriz. Escritos sobre literatura argentina. Edición a cargo de Sylvia Saítta, Siglo XXI Editores, 2007.

Sarlo Sabajanes, Beatriz. Juan María Gutiérrez: historiador y crítico de nuestra literatura. Editorial Escuela, 1967.

\section{Notas}

* Artículo de investigación

1 La sección incluye además un ensayo sobre el uso de la noción de 'desierto’ en la cultura argentina, que parte de la mención a Echeverría para atravesar rápidamente el siglo y llegar a los comienzos del XX y a Borges ("En el origen de la cultura argentina: Europa y el desierto", 1986); otro sobre la relación de Buenos Aires con Europa ("Buenos Aires, el exilio de Europa", 1999), y otro sobre la revista Contorno (“Los dos ojos de Contorno", 1983). Véase Sarlo (Escritos).

2 Tal como se indica en la portadilla, en la selección del libro para que fuera editado con apoyo económico del Fondo Nacional de las Artes, intervino un jurado integrado por Guillermo de Torre, José Bianco y Bernardo Canal Feijóo (Sarlo Juan María Gutiérrez). La edición fue de Editorial Escuela, una empresa ligada a la educación, como se infiere de su nombre, pero en cuyo catálogo la psicopedagogía convive con la psicología, el psicoanálisis y, aisladamente, con los estudios literarios. 
3 El interés de Sarlo, aunque con diversa manifestación, por el periodismo cultural, se observa tempranamente en la revista Los Libros (1969-1976), cuyo consejo de redacción integra a partir de principios de 1973 junto con Carlos Altamirano y Ricardo Piglia.

4 La colaboración entre Beatriz Sarlo y Carlos Altamirano abarca toda la década del ochenta y se completa con Conceptos de sociología literaria y con Literatura/Sociedad, publicados respectivamente por el Centro Editor de América Latina en 1980 y la editorial Hachette en 1983. Y ya en una dimensión político-cultural de largo plazo, que excede la actividad más coyuntural para la revista Los libros a comienzos de los setenta, esa colaboración se pone de manifiesto en la dirección de Punto de Vista, proyecto iniciado en 1978 y que concluye en 2008, pero del cual Altamirano se retira en 2004.

5 A propósito de esa reedición, la revista ., suplemento cultural del diario Clarín, publica una entrevista conjunta que les hace Héctor Pavón (18/7/2016) y que empieza, precisamente aunque contra lo que podría esperarse de ambos, con la afirmación de Sarlo: "Me quedo con Sarmiento", mientras Altamirano dice: "Yo, con Esteban Echeverría”.

6 Además de todos los fascículos mencionados, Sarlo colabora en la segunda edición de la Historia de la literatura argentina con un estudio sobre la poesía posmodernista de las primeras décadas del siglo XX en el que se dedica, entre otros, a Evaristo Carriego.

7 Cabe preguntarse por qué Sarlo se "saltea" la década del ochenta al pensar la modernización del campo cultural argentino y apuntar a un espacio autónomo para la literatura. Por un lado, es cierto que no hay un programa de modernización explícito, ni tampoco, en líneas generales, un gesto claramente autosuficiente en cuanto a una posible especificidad de lo literario y de la figura de escritor. Por otro lado, sin embargo, llama la atención que la importancia del profesionalismo detectado hacia el Centenario pase por alto la profesionalización que en los años ochenta empieza a estar disponible en la prensa periódica en tanto administradora de los bienes culturales. A la vez, el hecho de que el criterio de valoración estética sea cuestionable en gran parte de esas producciones para la prensa, en particular en los folletines, no explica del todo esa desatención, dado que Sarlo le dedicaría un libro en 1985 a los relatos sentimentales en El imperio de los sentimientos. Narraciones de circulación periódica en la Argentina, 1917-1927. Antes que con nociones como el valor estético, lo popular o lo moderno, considero que esa desatención se debe más bien a la ausencia, en los escritores de los ochenta, de postulaciones o autopostulaciones de corte literario que permitan detectar algún tipo de programa o de expectativa grupal sobre la constitución del campo cultural de esos años, cuando la literatura finalmente había dejado de estar inexorablemente atada a las urgencias de la política partidista.

8 Entre los libros de crítica literaria argentina, se recorta por su tema, en ese sentido, El género gauchesco. Un tratado sobre la patria, de Josefina Ludmer, también de 1988, igual que el de Sarlo.

\section{Licencia Creative Commons CC BY 4.0}

Cómo citar este artículo: Laera, Alejandra. "Fundaciones. Beatriz Sarlo en el siglo XIX". Cuadernos de Literatura, vol. 24, 2020. https://doi.org/10.11144/Javeriana.cl24.bses 